TRANSACTIONS OF THE

AMERICAN MATHEMATICAL SOCIETY

Volume 355, Number 7, Pages 2885-2903

S 0002-9947(03)03289-6

Article electronically published on March 14, 2003

\title{
WHEN ARE THE TANGENT SPHERE BUNDLES OF A RIEMANNIAN MANIFOLD REDUCIBLE?
}

\author{
E. BOECKX
}

\begin{abstract}
We determine all Riemannian manifolds for which the tangent sphere bundles, equipped with the Sasaki metric, are local or global Riemannian product manifolds.
\end{abstract}

\section{INTRODUCTION}

When studying the geometry of a Riemannian manifold $(M, g)$, it is often useful to relate it to the properties of its unit tangent sphere bundle $T_{1} M$. In earlier work, we have been primarily interested in the geometric properties of $T_{1} M$ when equipped with the Sasaki metric $g_{S}$. This is probably the simplest possible Riemannian metric on $T_{1} M$ and it is completely determined by the metric $g$ on the base manifold $M$. In this way, we have obtained a number of interesting characterizations of specific classes of Riemannian manifolds. We refer to [2], [5], [6], [7] and the references therein for examples of this. Also tangent sphere bundles $T_{r} M$ with radius $r$ different from 1 and equipped with the Sasaki metric have been studied recently ([9], [10]). The geometric properties of these Riemannian manifolds may change with the radius. See [9] for an example of this. Of course, other Riemannian metrics on the tangent bundle and on the tangent sphere bundles are possible. Of these, the Cheeger-Gromoll metric $g_{C G}$ may be the best known. However, for tangent sphere bundles, this specific metric yields nothing new, since $\left(T_{r} M, g_{C G}\right)$ is isometric to $\left(T_{r / \sqrt{1+r^{2}}} M, g_{S}\right)$. The isometry is given explicitly by $\phi: T_{r} M \rightarrow T_{r / \sqrt{1+r^{2}}} M:(x, u) \mapsto\left(x, u / \sqrt{1+r^{2}}\right)$.

It is an interesting geometric problem to determine when a tangent sphere bundle, which we always consider with the Sasaki metric in this paper, is reducible, i.e., when it is locally or globally isometric to a Riemannian product manifold. To our surprise, we could not find any results in the literature concerning this question. Nevertheless, knowledge about reducibility could help to deal with geometric questions about tangent sphere bundles. In 4 for instance, we use it in an essential way to determine all unit tangent sphere bundles that are semi-symmetric, i.e., for which the curvature tensor at each point is algebraically the same as that of some symmetric space. Actually, that problem was the inspiration for the present article. As concerns the local reducibility of tangent sphere bundles, we prove here the following.

Received by the editors November 11, 2002 and, in revised form, January 21, 2003.

2000 Mathematics Subject Classification. 53B20, 53C12, 53C20.

Key words and phrases. Tangent sphere bundle, Sasaki metric, reducibility, Clifford structures, foliations. 
Local Theorem. A tangent sphere bundle $\left(T_{r} M, g_{S}\right), r>0$, of a Riemannian manifold $\left(M^{n}, g\right), n \geq 2$, is locally reducible if and only if $(M, g)$ has a flat factor, i.e., $(M, g)$ is locally isometric to a product $\left(M^{\prime}, g^{\prime}\right) \times\left(\mathbb{R}^{k}, g_{0}\right)$ where $1 \leq k \leq n$ and $g_{0}$ denotes the standard Euclidean metric on $\mathbb{R}^{k}$.

The corresponding global version reads as follows:

Global Theorem. Let $\left(M^{n}, g\right), n \geq 3$, be a Riemannian manifold and suppose that $\left(T_{r} M, g_{S}\right)$ is a global Riemannian product. Then, $(M, g)$ is either flat or it is also a global Riemannian product, with a flat factor.

Conversely, if $(M, g)$ is a global product space $\left(M^{\prime}, g^{\prime}\right) \times\left(F^{k}, g_{0}\right)$ where $1 \leq k \leq n$ and $F$ is a connected and simply connected flat space, then $\left(T_{r} M, g_{S}\right)$ is a global Riemannian product, also with $\left(F, g_{0}\right)$ as a flat factor.

In view of the comments above, these results remain valid if we consider the tangent sphere bundles equipped with the Cheeger-Gromoll metric.

This article is organized as follows. After giving the necessary definitions and formulas concerning tangent sphere bundles, we show in Section 3 that only two types of decomposition for $T_{r} M$ are possible: a vertical and a diagonal one. The special form of the curvature of $\left(T_{r} M, g_{S}\right)$ for vertical vectors is crucial here. In particular, the same procedure does not go through for the tangent bundle $T M$. Section 4 deals with the diagonal case. We find that a diagonal decomposition gives rise to a Clifford representation via specific curvature operators. As a result, only base manifolds with dimension $2,3,4,7$ or 8 could possibly admit diagonal decompositions. The different dimensions are then handled separately. It turns out that diagonal decompositions can only be realized for a flat surface as base space. The general situation with a vertical decomposition is treated in Section 5 and leads to the Local Theorem above. The final section is devoted to global considerations.

\section{TANGENT SPHERE BUNDLES}

We first recall a few of the basic facts and formulas about the tangent sphere bundles of a Riemannian manifold. A more elaborate exposition and further references can be found in [5] and [9].

The tangent bundle $T M$ of a Riemannian manifold $(M, g)$ consists of pairs $(x, u)$ where $x$ is a point in $M$ and $u$ is a tangent vector to $M$ at $x$. The mapping $\pi: T M \rightarrow$ $M:(x, u) \mapsto x$ is the natural projection from $T M$ onto $M$. It is well known that the tangent space to $T M$ at a point $(x, u)$ splits into the direct sum of the vertical subspace $\operatorname{VT} M_{(x, u)}=\operatorname{ker} \pi_{* \mid(x, u)}$ and the horizontal subspace $\operatorname{HTM}_{(x, u)}$ with respect to the Levi-Civita connection $\nabla$ of $(M, g): T_{(x, u)} T M=V T M_{(x, u)} \oplus$ $\operatorname{HTM}_{(x, u)}$.

For $w \in T_{x} M$, there exists a unique horizontal vector $w^{h} \in H T M_{(x, u)}$ for which $\pi_{*}\left(w^{h}\right)=w$. It is called the horizontal lift of $w$ to $(x, u)$. There is also a unique vertical vector $w^{v} \in V T M_{(x, u)}$ for which $w^{v}(d f)=w(f)$ for all functions $f$ on $M$. It is called the vertical lift of $w$ to $(x, u)$. These lifts define isomorphisms between $T_{x} M$ and $\operatorname{HTM}_{(x, u)}$ and $V T M_{(x, u)}$, respectively. Hence, every tangent vector to $T M$ at $(x, u)$ can be written as the sum of a horizontal and a vertical lift of uniquely defined tangent vectors to $M$ at $x$. The horizontal (respectively vertical) lift of a vector field $X$ on $M$ to $T M$ is defined in the same way by lifting $X$ pointwise. Further, if $T$ is a tensor field of type $(1, s)$ on $M$ and $X_{1}, \ldots, X_{s-1}$ are vector fields on $M$, then we denote by $T\left(X_{1}, \ldots, u, \ldots, X_{s-1}\right)^{v}$ the vertical vector field on $T M$ 
which at $(x, w)$ takes the value $T\left(X_{1 x}, \ldots, w, \ldots, X_{s-1 x}\right)^{v}$, and similarly for the horizontal lift. In general, these are not the vertical or horizontal lifts of a vector field on $M$.

The Sasaki metric $g_{S}$ on $T M$ is completely determined by

$$
g_{S}\left(X^{h}, Y^{h}\right)=g_{S}\left(X^{v}, Y^{v}\right)=g(X, Y) \circ \pi, \quad g_{S}\left(X^{h}, Y^{v}\right)=0
$$

for vector fields $X$ and $Y$ on $M$.

Our interest lies in the tangent sphere bundle $T_{r} M$ of some positive radius $r$, which is a hypersurface of $T M$ consisting of all tangent vectors to $(M, g)$ of length $r$. It is given implicitly by the equation $g_{x}(u, u)=r^{2}$. A unit normal vector field $N$ to $T_{r} M$ is given by the vertical vector field $u^{v} / r$. We see that horizontal lifts to $(x, u) \in T_{r} M$ are tangent to $T_{r} M$, but vertical lifts in general are not. For that reason, we define the tangential lift $w^{t}$ of $w \in T_{x} M$ to $(x, u) \in T_{r} M$ by $w^{t}=w^{v}-\frac{1}{r} g(w, u) N$. Clearly, the tangent space to $T_{r} M$ at $(x, u)$ is spanned by horizontal and tangential lifts of tangent vectors to $M$ at $x$. One defines the tangential lift of a vector field $X$ on $M$ in the obvious way. For the sake of notational clarity, we will use $\bar{X}$ as a shorthand for $X-\frac{1}{r^{2}} g(X, u) u$. Then $X^{t}=\bar{X}^{v}$. Further, we denote by $V T_{r} M$ the $(n-1)$-dimensional distribution of vertical tangent vectors to $T_{r} M$.

If we consider $T_{r} M$ with the metric induced from the Sasaki metric $g_{S}$ of $T M$, also denoted by $g_{S}$, we turn $T_{r} M$ into a Riemannian manifold. Its Levi-Civita connection $\bar{\nabla}$ is described completely by

$$
\begin{aligned}
\bar{\nabla}_{X^{t}} Y^{t} & =-\frac{1}{r^{2}} g(Y, u) X^{t}, \\
\bar{\nabla}_{X^{t}} Y^{h} & =\frac{1}{2}(R(u, X) Y)^{h}, \\
\bar{\nabla}_{X^{h}} Y^{t} & =\left(\nabla_{X} Y\right)^{t}+\frac{1}{2}(R(u, Y) X)^{h}, \\
\bar{\nabla}_{X^{h}} Y^{h} & =\left(\nabla_{X} Y\right)^{h}-\frac{1}{2}(R(X, Y) u)^{t}
\end{aligned}
$$

for vector fields $X$ and $Y$ on $M$. Its Riemann curvature tensor $\bar{R}$ is given by

$$
\begin{aligned}
\bar{R}\left(X^{t}, Y^{t}\right) Z^{t}= & \frac{1}{r^{2}}\left(g(\bar{Y}, \bar{Z}) X^{t}-g(\bar{Z}, \bar{X}) Y^{t}\right) \\
\bar{R}\left(X^{t}, Y^{t}\right) Z^{h}= & (R(\bar{X}, \bar{Y}) Z)^{h}+\frac{1}{4}([R(u, X), R(u, Y)] Z)^{h}, \\
\bar{R}\left(X^{h}, Y^{t}\right) Z^{t}= & -\frac{1}{2}(R(\bar{Y}, \bar{Z}) X)^{h}-\frac{1}{4}(R(u, Y) R(u, Z) X)^{h}, \\
\bar{R}\left(X^{h}, Y^{t}\right) Z^{h}= & \frac{1}{2}(R(X, Z) \bar{Y})^{t}-\frac{1}{4}(R(X, R(u, Y) Z) u)^{t} \\
& +\frac{1}{2}\left(\left(\nabla_{X} R\right)(u, Y) Z\right)^{h}, \\
\bar{R}\left(X^{h}, Y^{h}\right) Z^{t}= & (R(X, Y) \bar{Z})^{t} \\
& +\frac{1}{4}(R(Y, R(u, Z) X) u-R(X, R(u, Z) Y) u)^{t} \\
& +\frac{1}{2}\left(\left(\nabla_{X} R\right)(u, Z) Y-\left(\nabla_{Y} R\right)(u, Z) X\right)^{h}, \\
\bar{R}\left(X^{h}, Y^{h}\right) Z^{h}= & (R(X, Y) Z)^{h}+\frac{1}{2}(R(u, R(X, Y) u) Z)^{h} \\
& -\frac{1}{4}(R(u, R(Y, Z) u) X-R(u, R(X, Z) u) Y)^{h} \\
& +\frac{1}{2}\left(\left(\nabla_{Z} R\right)(X, Y) u\right)^{t}
\end{aligned}
$$

for vector fields $X, Y$ and $Z$ on $M$. (See [9].) 


\section{TWO TYPES OF DECOMPOSITION}

Let $\left(M^{n}, g\right)$ be a Riemannian manifold of dimension $n \geq 2$ and suppose that its tangent sphere bundle $T_{r} M$ is (locally) reducible, i.e., $\left(T_{r} M, g_{S}\right) \simeq\left(M_{1}, g_{1}\right) \times$ $\left(M_{2}, g_{2}\right)$. A point $(x, u)$ in $T_{r} M$ corresponds to a couple $(p, q) \in M_{1} \times M_{2}$, and the tangent space $T_{(x, u)} T_{r} M$ can be identified with $T_{p} M_{1} \oplus T_{q} M_{2}$. In the sequel, we will write $T_{(x, u)} M_{1}$ and $T_{(x, u)} M_{2}$ for $T_{p} M_{1}$ and $T_{q} M_{2}$, considered as subspaces of $T_{(x, u)} T_{r} M$, in order not to make the notation too cumbersome.

Suppose first that, at a point $(x, u)$ of $T_{r} M$, the tangent space to one of the factors, say to $M_{1}$, contains a nonzero vertical vector $X^{t}, X \in T_{x} M$ and $X \perp u$. Since we have a Riemannian product, the curvature operator $\bar{R}(\mathbf{U}, \mathbf{V})$ preserves the tangent spaces to both factors for all vectors $\mathbf{U}$ and $\mathbf{V}$ tangent to $T_{r} M$. In particular, it follows that

$$
\bar{R}\left(Y^{t}, X^{t}\right) X^{t}=\frac{1}{r^{2}}\left(g(X, X) Y^{t}-g(X, Y) X^{t}\right) \in T_{(x, u)} M_{1}
$$

for all vectors $Y \in T_{x} M$. As a consequence, $V T_{r} M_{(x, u)} \subset T_{(x, u)} M_{1}$, and $M_{1}$ is at least $(n-1)$-dimensional. Hence, if at a point of $T_{r} M$ one of the factors contains a nonzero vertical vector, it contains the complete vertical distribution at that point. We call the decomposition vertical at $(x, u)$ in such a situation. Note that this is the case as soon as $\max \left\{\operatorname{dim} M_{1}, \operatorname{dim} M_{2}\right\}>n$. Indeed, if $\operatorname{dim} M_{1}>n$, then

$$
\begin{aligned}
\operatorname{dim}\left(V T_{r} M_{(x, u)} \cap T_{(x, u)} M_{1}\right)= & \operatorname{dim} V T_{r} M_{(x, u)}+\operatorname{dim} T_{(x, u)} M_{1} \\
& -\operatorname{dim}\left(V T_{r} M_{(x, u)}+T_{(x, u)} M_{1}\right) \\
> & (n-1)+n-(2 n-1)=0 .
\end{aligned}
$$

So, the only possibility for the decomposition not to be vertical at $(x, u)$ is that $\operatorname{dim} M_{1}=n, \operatorname{dim} M_{2}=n-1$ (or conversely) and neither factor is tangent to a vertical vector. We call this a diagonal decomposition at $(x, u)$.

The major part of the sequel will be devoted to the diagonal case. Using a purely infinitesimal (i.e., pointwise) approach, we show that a diagonal decomposition is only possible in one specific situation. Afterwards, we study the case of a vertical decomposition.

\section{Diagonal Decomposition}

4.1. A suitable basis. In this section, we consider a diagonal decomposition $T_{r} M \simeq M_{1} \times M_{2}$ at $(x, u)$ with $\operatorname{dim} M_{1}=n$ and $\operatorname{dim} M_{2}=n-1$. For dimensional reasons, we have

$$
\operatorname{dim}\left(T_{(x, u)} M_{1} \cap \operatorname{HTM}_{(x, u)}\right)>0 .
$$

Let $X_{n} \in T_{x} M$ be a unit vector such that $X_{n}{ }^{h}$ is tangent to $M_{1}$ at $(x, u)$ and extend it to an orthonormal basis $\left\{X_{1}, \ldots, X_{n}\right\}$ of $T_{x} M$. If $\pi_{*(x, u)}\left(T_{(x, u)} M_{1}\right) \neq$ $T_{x} M$, then there must be a vertical vector tangent to $M_{1}$ at $(x, u)$, contrary to the hypothesis. Hence, there exist well-defined vectors $Y_{1}, \ldots, Y_{n-1}$ orthogonal to $u$ for which $X_{1}{ }^{h}+Y_{1}{ }^{t}, \ldots, X_{n-1}{ }^{h}+Y_{n-1}{ }^{t}$ and $X_{n}{ }^{h}$ are tangent to $M_{1}$ at $(x, u)$. Clearly, they form a basis for $T_{(x, u)} M_{1}$, though not in general an orthonormal one. Moreover, $\left\{Y_{1}, \ldots, Y_{n-1}, u\right\}$ is a basis for $T_{x} M$ too. Otherwise, there would exist a nonzero vector $Y \in T_{x} M$, orthogonal to $u$ and to $Y_{i}, i=1, \ldots, n-1$. But then $Y^{t}$ would be orthogonal to $X_{n}{ }^{h}$ and to $X_{i}{ }^{h}+Y_{i}{ }^{t}, i=1, \ldots, n-1$, and hence 
would belong to $\left(T_{(x, u)} M_{1}\right)^{\perp}=T_{(x, u)} M_{2}$, contrary to the hypothesis that $M_{2}$ has no vertical tangent vector.

Next, consider the $(n-1) \times(n-1)$ matrix $\alpha=\left(g_{x}\left(Y_{i}, Y_{j}\right)\right)_{i, j=1, \ldots, n-1}$. Since this matrix is symmetric and positive definite, it can be diagonalized by a suitable orthogonal transformation:

$$
P \alpha P^{t}=\operatorname{diag}\left(\lambda_{1}^{2}, \ldots, \lambda_{n-1}^{2}\right)
$$

where $P=\left(p_{i j}\right) \in O(n-1)$ and $\lambda_{i}>0$ for $i=1, \ldots, n-1$. If we put

$$
\tilde{X}_{i}=\sum_{j=1}^{n-1} p_{i j} X_{j}, \quad \tilde{Y}_{i}=\frac{1}{\lambda_{i}} \sum_{j=1}^{n-1} p_{i j} Y_{j}
$$

for $i=1, \ldots, n-1$, then both $\left\{\tilde{X}_{1}, \ldots, \tilde{X}_{n-1}, X_{n}\right\}$ and $\left\{\tilde{Y}_{1}, \ldots, \tilde{Y}_{n-1}, u / r\right\}$ are orthonormal bases for $T_{x} M$. Further, the vectors

$$
\tilde{X}_{i}^{h}+\lambda_{i} \tilde{Y}_{i}^{t}=\sum_{j=1}^{n-1} p_{i j}\left(X_{j}{ }^{h}+Y_{j}^{t}\right), \quad i=1, \ldots, n-1,
$$

together with $X_{n}{ }^{h}$ span the tangent space to $M_{1}$ at $(x, u)$ and these vectors are pairwise orthogonal. The tangent space to $M_{2}$ at $(x, u)$ is then spanned by the orthogonal vectors

$$
\lambda_{i} \tilde{X}_{i}^{h}-\tilde{Y}_{i}^{t}, \quad i=1, \ldots, n-1 .
$$

Finally, we show that all the numbers $\lambda_{i}$ are equal. To do this, we use that $g_{S}(\bar{R}(\mathbf{U}, \mathbf{V}) \mathbf{W}, \mathbf{T})=0$ at $(x, u)$ as soon as one of the vectors involved is tangent to $M_{1}$ and another one is tangent to $M_{2}$. In particular, for all $i, j, k, l=1, \ldots, n-1$, it follows that

$$
0=g_{S}\left(\bar{R}\left(\tilde{X}_{j}^{h}+\lambda_{j} \tilde{Y}_{j}^{t}, \tilde{Y}_{k}^{t}\right)\left(\lambda_{i} \tilde{X}_{i}^{h}-\tilde{Y}_{i}^{t}\right), \tilde{Y}_{l}^{t}\right) .
$$

Using the expressions (2) for the curvature tensor $\bar{R}$ of $\left(T_{r} M, g_{S}\right)$, this leads to the condition

$$
0=\lambda_{i}\left(2 g\left(R\left(\tilde{X}_{j}, \tilde{X}_{i}\right) \tilde{Y}_{k}, \tilde{Y}_{l}\right)-g\left(R\left(u, \tilde{Y}_{l}\right) \tilde{X}_{j}, R\left(u, \tilde{Y}_{k}\right) \tilde{X}_{i}\right)\right)-\frac{4 \lambda_{j}}{r^{2}}\left(\delta_{i k} \delta_{j l}-\delta_{i j} \delta_{k l}\right) .
$$

Switching the indices $i$ and $j$, as well as $k$ and $l$, we find

$$
0=\lambda_{j}\left(2 g\left(R\left(\tilde{X}_{i}, \tilde{X}_{j}\right) \tilde{Y}_{l}, \tilde{Y}_{k}\right)-g\left(R\left(u, \tilde{Y}_{k}\right) \tilde{X}_{i}, R\left(u, \tilde{Y}_{l}\right) \tilde{X}_{j}\right)\right)-\frac{4 \lambda_{i}}{r^{2}}\left(\delta_{j l} \delta_{i k}-\delta_{j i} \delta_{l k}\right) .
$$

Using the symmetries of the curvature tensor, it then easily follows that $\lambda_{i}{ }^{2}=\lambda_{j}{ }^{2}$ or $\lambda_{i}=\lambda_{j}$.

Summarizing, we have

Lemma. If $T_{r} M \simeq M_{1} \times M_{2}$ is a diagonal decomposition at $(x, u)$ with $\operatorname{dim} M_{1}=$ $n$ and $\operatorname{dim} M_{2}=n-1$, then there exist orthonormal bases $\left\{X_{1}, \ldots, X_{n}\right\}$ and $\left\{Y_{1}, \ldots, Y_{n-1}, u / r\right\}$ of $T_{x} M$ and $\lambda>0$, such that an orthogonal basis for $T_{(x, u)} M_{1}$ is given by

$$
X_{1}^{h}+\lambda Y_{1}^{t}, \ldots, X_{n-1}{ }^{h}+\lambda Y_{n-1}{ }^{t}, X_{n}{ }^{h}
$$

and an orthogonal basis for $T_{(x, u)} M_{2}$ is given by

$$
\lambda X_{1}^{h}-Y_{1}^{t}, \ldots, \lambda X_{n-1}^{h}-Y_{n-1}{ }^{t} .
$$


Remark 1. The number $\lambda$ has a clear geometric meaning. Take a nonzero vertical vector $\mathbf{U}$ at $(x, u): \mathbf{U}=\sum_{i=1}^{n-1} \alpha_{i} Y_{i}^{t}$ and a nonzero vector $\mathbf{V}$ tangent to $M_{2}$ at $(x, u)$ : $\mathbf{V}=\sum_{i=1}^{n-1} \beta_{i}\left(\lambda X_{i}{ }^{h}-Y_{i}{ }^{t}\right)$. The angle between the two vectors has cosine given by

$$
\cos (\widehat{\mathbf{U V}})=\frac{-\sum \alpha_{i} \beta_{i}}{\sqrt{\sum \alpha_{i}^{2}} \sqrt{\sum \beta_{i}^{2}} \sqrt{1+\lambda^{2}}} .
$$

By the Cauchy-Schwarz inequality, we have

$$
-\frac{1}{\sqrt{1+\lambda^{2}}} \leq \cos (\widehat{\mathbf{U V}}) \leq \frac{1}{\sqrt{1+\lambda^{2}}}
$$

with equality if and only if $\left(\alpha_{1}, \ldots, \alpha_{n-1}\right)$ and $\left(\beta_{1}, \ldots, \beta_{n-1}\right)$ are proportional. We conclude that the angle $\theta$ between $V T_{r} M_{(x, u)}$ and $T_{(x, u)} M_{2}$ is such that $\cos \theta=$ $1 / \sqrt{1+\lambda^{2}}$ or $\tan \theta=\lambda$. So, $\lambda$ determines the angle between $V T_{r} M$ and $M_{2}$ at $(x, u)$ (and hence also between $V T_{r} M$ and $M_{1}$ at that point).

Remark 2. Actually, we can give a stronger formulation of the lemma. To see this, consider the mapping $\pi_{1}: T_{(x, u)} M_{1} \rightarrow V T_{r} M_{(x, u)}: X^{h}+Y^{t} \mapsto Y^{t}$. Clearly, this mapping is linear and one-to-one on $\left(X_{n}{ }^{h}\right)^{\perp}$. We restrict $\pi_{1}$ to $\left(X_{n}{ }^{h}\right)^{\perp} \cap T_{(x, u)} M_{1}$ and define the linear mapping

$$
A: u^{\perp} \rightarrow X_{n}^{\perp}: Y \mapsto \lambda \pi_{*(x, u)}\left(\pi_{1}^{-1} Y^{t}\right)
$$

where, as before, $\pi: T_{r} M \rightarrow M$ is the natural projection map. Since

$$
A Y_{i}=\lambda \pi_{*(x, u)}\left(\pi_{1}^{-1} Y_{i}^{t}\right)=\lambda \pi_{*(x, u)}\left(\left(X_{i}^{h}+\lambda Y_{i}^{t}\right) / \lambda\right)=X_{i},
$$

the map $A$ is an isometry from $u^{\perp}$ to $X_{n}{ }^{\perp}$. It associates to a vector $X$, orthogonal to $X_{n}$, the unique vector $Y$, orthogonal to $u$, such that $X^{h}+\lambda Y^{t}$ is tangent to $M_{1}$ at $(x, u)$ (or such that $\lambda X^{h}-Y^{t}$ is tangent to $M_{2}$ at $(x, u)$ ). So, in the lemma, we can actually choose an arbitrary orthonormal basis $\left\{X_{1}, \ldots, X_{n-1}\right\}$ of $X_{n} \perp$ (or, alternatively, an arbitrary orthonormal basis $\left\{Y_{1}, \ldots, Y_{n-1}\right\}$ of $\left.u^{\perp}\right)$. We will use this possibility in the subsequent subsections. The vectors $X_{n}$ (up to sign) and $u$, on the other hand, are determined geometrically.

4.2. Curvature conditions. Since $\left(T_{r} M, g_{S}\right)$ is a (local) Riemannian product, all the expressions of the form $\bar{R}(\mathbf{U}, \mathbf{V}) \mathbf{W}$ are zero when $\mathbf{U}$ is tangent to $M_{1}$ and $\mathbf{W}$ is tangent to $M_{2}$ at $(x, u)$. Using the curvature formulas (2), this leads to a number of curvature conditions for the manifold $M$. We list some of these now. From now on, indices $i, j, k$ and $l$ belong to $\{1, \ldots, n-1\}$ unless stated otherwise.

The tangential and horizontal components of $\bar{R}\left(X_{n}{ }^{h}, Y_{j}{ }^{t}\right)\left(\lambda X_{k}{ }^{h}-Y_{k}{ }^{t}\right)$ give rise to

$$
\begin{aligned}
& 2 R\left(X_{n}, X_{k}\right) Y_{j}-\frac{2}{r^{2}} g\left(R\left(X_{n}, X_{k}\right) Y_{j}, u\right) u=R\left(X_{n}, R\left(u, Y_{j}\right) X_{k}\right) u \\
& 2 \lambda\left(\nabla_{X_{n}} R\right)\left(u, Y_{j}\right) X_{k}=-2 R\left(Y_{j}, Y_{k}\right) X_{n}-R\left(u, Y_{j}\right) R\left(u, Y_{k}\right) X_{n}
\end{aligned}
$$

while $\bar{R}\left(X_{n}{ }^{h}, X_{j}{ }^{h}\right)\left(\lambda X_{k}{ }^{h}-Y_{k}{ }^{t}\right)=0$ leads to

$$
\begin{aligned}
2 \lambda\left(\nabla_{X_{k}} R\right)\left(X_{n}, X_{j}\right) u=4 R\left(X_{n}, X_{j}\right) Y_{k}-\frac{4}{r^{2}} g\left(R\left(X_{n}, X_{j}\right) Y_{k}, u\right) u & \\
& +R\left(X_{j}, R\left(u, Y_{k}\right) X_{n}\right) u-R\left(X_{n}, R\left(u, Y_{k}\right) X_{j}\right) u \\
2\left(\left(\nabla_{X_{n}} R\right)\right. & \left.\left(u, Y_{k}\right) X_{j}-\left(\nabla_{X_{j}} R\right)\left(u, Y_{k}\right) X_{n}\right) \\
= & 4 \lambda R\left(X_{n}, X_{j}\right) X_{k}+2 \lambda R\left(u, R\left(X_{n}, X_{j}\right) u\right) X_{k} \\
& -\lambda R\left(u, R\left(X_{j}, X_{k}\right) u\right) X_{n}+\lambda R\left(u, R\left(X_{n}, X_{k}\right) u\right) X_{j} .
\end{aligned}
$$


Considering $\bar{R}\left(X_{i}^{h}+\lambda Y_{i}^{t}, Y_{j}^{t}\right)\left(\lambda X_{k}^{h}-Y_{k}^{t}\right)=0$, we obtain

$$
\begin{aligned}
& 2 R\left(X_{i}, X_{k}\right) Y_{j}-\frac{2}{r^{2}} g\left(R\left(X_{i}, X_{k}\right) Y_{j}, u\right) u \\
& \quad=R\left(X_{i}, R\left(u, Y_{j}\right) X_{k}\right) u+\frac{4}{r^{2}}\left(\delta_{j k} Y_{i}-\delta_{i k} Y_{j}\right), \\
& 2 \lambda\left(\nabla_{X_{i}} R\right)\left(u, Y_{j}\right) X_{k}+2 R\left(Y_{j}, Y_{k}\right) X_{i}+R\left(u, Y_{j}\right) R\left(u, Y_{k}\right) X_{i} \\
& \quad+\lambda^{2}\left(4 R\left(Y_{i}, Y_{j}\right) X_{k}+R\left(u, Y_{i}\right) R\left(u, Y_{j}\right) X_{k}-R\left(u, Y_{j}\right) R\left(u, Y_{i}\right) X_{k}\right)=0 .
\end{aligned}
$$

Finally, from $\bar{R}\left(X_{i}{ }^{h}+\lambda Y_{i}{ }^{t}, X_{j}{ }^{h}\right)\left(\lambda X_{k}{ }^{h}-Y_{k}{ }^{t}\right)=0$, we derive

$$
\begin{aligned}
& 2 \lambda\left(\nabla_{X_{k}} R\right)\left(X_{i}, X_{j}\right) u-4 R\left(X_{i}, X_{j}\right) Y_{k}+\frac{4}{r^{2}} g\left(R\left(X_{i}, X_{j}\right) Y_{k}, u\right) u \\
& \quad-R\left(X_{j}, R\left(u, Y_{k}\right) X_{i}\right) u+R\left(X_{i}, R\left(u, Y_{k}\right) X_{j}\right) u-2 \lambda^{2} R\left(X_{j}, X_{k}\right) Y_{i} \\
& \quad+\frac{2 \lambda^{2}}{r^{2}} g\left(R\left(X_{j}, X_{k}\right) Y_{i}, u\right) u+\lambda^{2} R\left(X_{j}, R\left(u, Y_{i}\right) X_{k}\right) u=0, \\
& 4 \lambda R\left(X_{i}, X_{j}\right) X_{k}+2 \lambda R\left(u, R\left(X_{i}, X_{j}\right) u\right) X_{k}-\lambda R\left(u, R\left(X_{j}, X_{k}\right) u\right) X_{i} \\
& \quad+\lambda R\left(u, R\left(X_{i}, X_{k}\right) u\right) X_{j}-2\left(\nabla_{X_{i}} R\right)\left(u, Y_{k}\right) X_{j}+2\left(\nabla_{X_{j}} R\right)\left(u, Y_{k}\right) X_{i} \\
& \quad-2 \lambda^{2}\left(\nabla_{X_{j}} R\right)\left(u, Y_{i}\right) X_{k}-2 \lambda R\left(Y_{i}, Y_{k}\right) X_{j}-\lambda R\left(u, Y_{i}\right) R\left(u, Y_{k}\right) X_{j}=0 .
\end{aligned}
$$

These conditions can be rewritten in an easier form. To start, we take the inner product of (3) with $Y_{l}$. This gives

$$
\begin{aligned}
2 g\left(R\left(X_{n}, X_{k}\right) Y_{j}, Y_{l}\right) & =g\left(R\left(X_{n}, R\left(u, Y_{j}\right) X_{k}\right) u, Y_{l}\right) \\
& =g\left(R\left(u, Y_{l}\right) X_{n}, R\left(u, Y_{j}\right) X_{k}\right) \\
& =-g\left(R\left(u, Y_{j}\right) R\left(u, Y_{l}\right) X_{n}, X_{k}\right) .
\end{aligned}
$$

This is equivalent to

$$
2 R\left(Y_{j}, Y_{l}\right) X_{n}+R\left(u, Y_{j}\right) R\left(u, Y_{l}\right) X_{n}=-g\left(R\left(u, Y_{j}\right) X_{n}, R\left(u, Y_{l}\right) X_{n}\right) X_{n} .
$$

By interchanging the indices $j$ and $l$ in this expression and adding both formulas, respectively subtracting them, we get

$$
\begin{aligned}
& R\left(u, Y_{j}\right) R\left(u, Y_{l}\right) X_{n}+R\left(u, Y_{l}\right) R\left(u, Y_{j}\right) X_{n} \\
& \quad=-2 g\left(R\left(u, Y_{j}\right) X_{n}, R\left(u, Y_{l}\right) X_{n}\right) X_{n}, \\
& R\left(u, Y_{j}\right) R\left(u, Y_{l}\right) X_{n}-R\left(u, Y_{l}\right) R\left(u, Y_{j}\right) X_{n}=4 R\left(Y_{l}, Y_{j}\right) X_{n} .
\end{aligned}
$$

Substituting (11) in (4), we find the simpler form

$$
2 \lambda\left(\nabla_{X_{n}} R\right)\left(u, Y_{j}\right) X_{k}=g\left(R\left(u, Y_{j}\right) X_{n}, R\left(u, Y_{k}\right) X_{n}\right) X_{n} .
$$

Next, we substitute (3) in (5) to obtain

$$
2 \lambda\left(\nabla_{X_{k}} R\right)\left(X_{n}, X_{j}\right) u=R\left(X_{n}, R\left(u, Y_{k}\right) X_{j}\right) u+R\left(X_{j}, R\left(u, Y_{k}\right) X_{n}\right) u .
$$

Taking the inner product with $Y_{l}$, we get

$$
\begin{aligned}
2 \lambda & g\left(\left(\nabla_{X_{k}} R\right)\left(X_{n}, X_{j}\right) u, Y_{l}\right) \\
\quad & =g\left(R\left(X_{n}, R\left(u, Y_{k}\right) X_{j}\right) u, Y_{l}\right)+g\left(R\left(X_{j}, R\left(u, Y_{k}\right) X_{n}\right) u, Y_{l}\right) \\
& =g\left(R\left(u, Y_{l}\right) X_{n}, R\left(u, Y_{k}\right) X_{j}\right)+g\left(R\left(u, Y_{l}\right) X_{j}, R\left(u, Y_{k}\right) X_{n}\right) \\
& =-g\left(R\left(u, Y_{k}\right) R\left(u, Y_{l}\right) X_{n}, X_{j}\right)-g\left(R\left(u, Y_{l}\right) R\left(u, Y_{k}\right) X_{n}, X_{j}\right) \\
& =0
\end{aligned}
$$


by (12). Hence,

$$
\left(\nabla_{X_{k}} R\right)\left(X_{n}, X_{j}\right) u=0 \quad \text { or, equivalently, } \quad\left(\nabla_{X_{k}} R\right)\left(u, Y_{l}\right) X_{n}=0 .
$$

Substituting (14) and (15) in (6), we find

$$
\begin{aligned}
\frac{1}{\lambda^{2}} & g\left(R\left(u, Y_{j}\right) X_{n}, R\left(u, Y_{k}\right) X_{n}\right) X_{n} \\
= & R\left(X_{n}, X_{j}\right) X_{k}+2 R\left(u, R\left(X_{n}, X_{j}\right) u\right) X_{k} \\
& \quad-R\left(u, R\left(X_{j}, X_{k}\right) u\right) X_{n}+R\left(u, R\left(X_{n}, X_{k}\right) u\right) X_{j} .
\end{aligned}
$$

In order to rewrite (7), we proceed as with (3)): we take the inner product with $Y_{l}$, and we use curvature properties to obtain

$$
2 R\left(Y_{j}, Y_{l}\right) X_{i}+R\left(u, Y_{j}\right) R\left(u, Y_{l}\right) X_{i}=\frac{4}{r^{2}}\left(\delta_{i l} X_{j}-\delta_{j l} X_{i}\right) .
$$

(Note that we also need (11) to know that the left-hand side in (17) is orthogonal to $X_{n}$.) Again switching the indices $j$ and $l$ and adding and subtracting the two formulas, we get

$$
\begin{aligned}
& R\left(u, Y_{j}\right) R\left(u, Y_{l}\right) X_{i}+R\left(u, Y_{l}\right) R\left(u, Y_{j}\right) X_{i}=\frac{4}{r^{2}}\left(\delta_{i l} X_{j}-2 \delta_{j l} X_{i}+\delta_{i j} X_{l}\right) \\
& R\left(u, Y_{j}\right) R\left(u, Y_{l}\right) X_{i}-R\left(u, Y_{l}\right) R\left(u, Y_{j}\right) X_{i}=4 R\left(Y_{l}, Y_{j}\right) X_{i}+\frac{4}{r^{2}}\left(\delta_{i l} X_{j}-\delta_{i j} X_{l}\right)
\end{aligned}
$$

Substituting (17) and (19) in (8), this reduces to

$$
\lambda\left(\nabla_{X_{i}} R\right)\left(u, Y_{j}\right) X_{k}=\frac{2\left(\lambda^{2}-1\right)}{r^{2}}\left(\delta_{i k} X_{j}-\delta_{j k} X_{i}\right),
$$

or equivalently, via (15), to

$$
\lambda\left(\nabla_{X_{i}} R\right)\left(X_{k}, X_{l}\right) u=\frac{2\left(\lambda^{2}-1\right)}{r^{2}}\left(\delta_{i k} Y_{l}-\delta_{i l} Y_{k}\right) .
$$

It is now easily verified that (9) is a consequence of the above formulas. As to (10), using (17) and (201), it simplifies to

$$
\begin{gathered}
4 R\left(X_{i}, X_{j}\right) X_{k}+2 R\left(u, R\left(X_{i}, X_{j}\right) u\right) X_{k}-R\left(u, R\left(X_{j}, X_{k}\right) u\right) X_{i} \\
\quad+R\left(u, R\left(X_{i}, X_{k}\right) u\right) X_{j}=\frac{4\left(\lambda^{4}-\lambda^{2}+1\right)}{\lambda^{2} r^{2}}\left(\delta_{j k} X_{i}-\delta_{i k} X_{j}\right) .
\end{gathered}
$$

In the rest of this section, we will only need the formulas (12), (13), (16), (18), (19) and (22).

4.3. Clifford structures. Putting $j=l$ in (12) and (18), we see that

$$
\begin{aligned}
R\left(u, Y_{j}\right)^{2} X_{j} & =0 \\
R\left(u, Y_{j}\right)^{2} X_{i} & =-\frac{4}{r^{2}} X_{i}, \quad i \neq j, \\
R\left(u, Y_{j}\right)^{2} X_{n} & =-\left|R\left(u, Y_{j}\right) X_{n}\right|^{2} X_{n} .
\end{aligned}
$$

Since $R\left(u, Y_{j}\right)$ is a skew-symmetric operator, the nonzero eigenvalues of $R\left(u, Y_{j}\right)^{2}$ must have even multiplicity. Hence,

- if $n$ is even, the eigenvalue $-4 / r^{2}$ has even multiplicity $n-2$ on $\left\{X_{j}, X_{n}\right\}^{\perp}$. Hence, the eigenvalue corresponding to $X_{n}$ must be zero. This implies that $R\left(u, Y_{j}\right) X_{n}=0$ for $j=1, \ldots, n-1$. By (13), also $R\left(Y_{j}, Y_{k}\right) X_{n}=0$ for $j, k=1, \ldots, n-1$. We conclude that $X_{n}$ belongs to the nullity distribution 
of the curvature tensor $R_{x}$. In this case, the conditions (12), (13) and (16) are trivially satisfied;

- if $n$ is odd, the eigenvalue $-4 / r^{2}$ has odd multiplicity $n-2$ on $\left\{X_{j}, X_{n}\right\}^{\perp}$. So, the eigenvalue corresponding to $X_{n}$ must be $-4 / r^{2}$ as well. Hence, it follows that $\left|R\left(u, Y_{j}\right) X_{n}\right|^{2}=4 / r^{2}$ for $j=1, \ldots, n-1$. By Remark 2, we even have $\left|R(u, Y) X_{n}\right|^{2}=4 / r^{2}$ for every unit vector $Y$ orthogonal to $u$. Polarizing this identity, we obtain $g\left(R(u, Y) X_{n}, R(u, Z) X_{n}\right)=\left(4 / r^{2}\right) g(Y, Z)$ for all vectors $Y$ and $Z$ orthogonal to $u$. In particular, the right-hand side of (12) equals $-\left(8 \delta_{j l} / r^{2}\right) X_{n}$. In this case, conditions (12) and (13) are included in (18) and (19) if we allow the index $i$ to be $n$.

Next, we put $i=j \neq l$ in (18). Since $R\left(u, Y_{j}\right) X_{j}=0$ (this follows from $\left.R\left(u, Y_{j}\right)^{2} X_{j}=0\right)$, we obtain $R\left(u, Y_{j}\right) R\left(u, Y_{l}\right) X_{j}=\left(4 / r^{2}\right) X_{l}$. Applying the operator $R\left(u, Y_{j}\right)$ on both sides, we have

$$
\begin{aligned}
\frac{4}{r^{2}} R\left(u, Y_{j}\right) X_{l}= & R\left(u, Y_{j}\right)^{2} R\left(u, Y_{l}\right) X_{j} \\
= & -\frac{4}{r^{2}}\left(R\left(u, Y_{l}\right) X_{j}-g\left(R\left(u, Y_{l}\right) X_{j}, X_{n}\right) X_{n}\right) \\
& -g\left(R\left(u, Y_{l}\right) X_{j}, X_{n}\right)\left|R\left(u, Y_{j}\right) X_{n}\right|^{2} X_{n}
\end{aligned}
$$

or, equivalently,

$$
4\left(R\left(u, Y_{j}\right) X_{l}+R\left(u, Y_{l}\right) X_{j}\right)=\left(4-r^{2}\left|R\left(u, Y_{j}\right) X_{n}\right|^{2}\right) g\left(R\left(u, Y_{l}\right) X_{j}, X_{n}\right) X_{n} .
$$

Since the right-hand side of this expression vanishes both when $n$ is odd and when $n$ is even, we conclude

$$
R\left(u, Y_{j}\right) X_{l}+R\left(u, Y_{l}\right) X_{j}=0
$$

for $j, l=1, \ldots, n-1$.

We are now ready to discover Clifford representations in our formulas, in particular in (12) and (18). First, consider the case when $n$ is even. For $j=1, \ldots, n-1$, define the operators $\mathbf{R}_{i}$ acting on $V^{n}=T_{x} M$ by

$$
\mathbf{R}_{i}=\frac{r}{2} R\left(u, Y_{i}\right)-\left\langle X_{n}, \cdot\right\rangle X_{i}+\left\langle X_{i}, \cdot\right\rangle X_{n}
$$

where $\langle\cdot\rangle=,g_{x}$. In particular, it follows that $\mathbf{R}_{i} X_{i}=X_{n}, \mathbf{R}_{i} X_{n}=-X_{i}$ and $\mathbf{R}_{i} X_{j}=(r / 2) R\left(u, Y_{i}\right) X_{j}, j \neq i$. Clearly, $\mathbf{R}_{i}$ is a skew-symmetric operator and $\mathbf{R}_{i}{ }^{2}=-\mathrm{id}$. 
For $i \neq j \neq k \neq i$, we calculate:

$$
\begin{aligned}
&\left(\mathbf{R}_{i} \circ\right.\left.\mathbf{R}_{j}+\mathbf{R}_{j} \circ \mathbf{R}_{i}\right) X_{n}=-\mathbf{R}_{i} X_{j}-\mathbf{R}_{j} X_{i} \\
&=\left.-\frac{r}{2}\left(R\left(u, Y_{i}\right) X_{j}+R\left(u, Y_{j}\right) X_{i}\right)=0, \quad \text { by (23) }\right) \\
&\left(\mathbf{R}_{i} \circ\right.\left.\mathbf{R}_{j}+\mathbf{R}_{j} \circ \mathbf{R}_{i}\right) X_{i}=\mathbf{R}_{i}\left(\frac{r}{2} R\left(u, Y_{j}\right) X_{i}\right)+\mathbf{R}_{j} X_{n} \\
&=\left.\frac{r^{2}}{4} R\left(u, Y_{i}\right) R\left(u, Y_{j}\right) X_{i}-X_{j}=0 \quad \text { by (18) }\right) \\
&\left(\mathbf{R}_{i} \circ \mathbf{R}_{j}+\mathbf{R}_{j} \circ \mathbf{R}_{i}\right) X_{k}=\mathbf{R}_{i}\left(\frac{r}{2} R\left(u, Y_{j}\right) X_{k}\right)+\mathbf{R}_{j}\left(\frac{r}{2} R\left(u, Y_{i}\right) X_{k}\right) \\
&=\frac{r^{2}}{4}\left(R\left(u, Y_{i}\right)\left\{R\left(u, Y_{j}\right) X_{k}-g\left(R\left(u, Y_{j}\right) X_{k}, X_{i}\right) X_{i}\right\}\right. \\
&\left.\quad+R\left(u, Y_{j}\right)\left\{R\left(u, Y_{i}\right) X_{k}-g\left(R\left(u, Y_{i}\right) X_{k}, X_{j}\right) X_{j}\right\}\right) \\
&+\frac{r}{2}\left(g\left(R\left(u, Y_{j}\right) X_{k}, X_{i}\right)+g\left(R\left(u, Y_{i}\right) X_{k}, X_{j}\right)\right) X_{n} \\
&=\frac{r^{2}}{4}\left(R\left(u, Y_{i}\right) R\left(u, Y_{j}\right) X_{k}+R\left(u, Y_{j}\right) R\left(u, Y_{i}\right) X_{k}\right) \\
& \quad-\frac{r}{2} g\left(R\left(u, Y_{j}\right) X_{i}+R\left(u, Y_{i}\right) X_{j}, X_{k}\right) X_{n} \\
&=0 \quad(\text { by (18) and (23) }) .
\end{aligned}
$$

So, for $i, j=1, \ldots, n-1$, the operators $\mathbf{R}_{i}$ satisfy

$$
\mathbf{R}_{i} \circ \mathbf{R}_{j}+\mathbf{R}_{j} \circ \mathbf{R}_{i}=-2 \delta_{i j} \text { id }
$$

and they correspond to a Clifford representation of an $(n-1)$-dimensional Clifford algebra on an $n$-dimensional vector space.

It is well known (see, e.g., 1] or 3]) that a given real Clifford algebra, say of dimension $m$, has only one (if $m \neq \equiv 3(\bmod 4))$ or two $($ if $m \equiv 3(\bmod 4))$ irreducible representations and that the dimension $n_{0}$ of the corresponding irreducible Clifford module is completely determined by $m$. This relationship is given in the following table.

\begin{tabular}{|l|cccccccc|}
\hline$m$ & $8 p$ & $8 p+1$ & $8 p+2$ & $8 p+3$ & $8 p+4$ & $8 p+5$ & $8 p+6$ & $8 p+7$ \\
\hline$n_{0}$ & $2^{4 p}$ & $2^{4 p+1}$ & $2^{4 p+2}$ & $2^{4 p+2}$ & $2^{4 p+3}$ & $2^{4 p+3}$ & $2^{4 p+3}$ & $2^{4 p+3}$ \\
\hline
\end{tabular}

For a reducible Clifford module, the dimension is a multiple $k n_{0}$ of the number $n_{0}$ corresponding to the appropriate Clifford algebra.

In the present situation, we have $m=n-1$ and $k n_{0}=n$ for even $n$. Therefore:

- if $n=8 p: 8 p=k 2^{4 p-1}$ and hence $p=1, k=1$ and $n=8$;

- if $n=8 p+2: 8 p+2=k 2^{4 p+1}$ and hence $p=0, k=1$ and $n=2$;

- if $n=8 p+4: 8 p+4=k 2^{4 p+2}$ and hence $p=0, k=1$ and $n=4$;

- if $n=8 p+6: 8 p+6=k 2^{4 p+3}$, which has no solutions.

Next, suppose that $n$ is odd. Now, we define operators $\mathbf{R}_{i}, i=1, \ldots, n-1$, acting on $V^{n+1}=T_{x} M \oplus \mathbb{R} X_{0}$ by

$$
\mathbf{R}_{i}=\frac{r}{2} R\left(u, Y_{i}\right)-\left\langle X_{0}, \cdot\right\rangle X_{i}+\left\langle X_{i}, \cdot\right\rangle X_{0}
$$


where $\langle\cdot, \cdot\rangle=g_{x} \oplus g_{0}$ with $g_{0}\left(a X_{0}, b X_{0}\right)=a b$. Precisely as before, we show that $\mathbf{R}_{i} \circ \mathbf{R}_{j}+\mathbf{R}_{j} \circ \mathbf{R}_{i}=-2 \delta_{i j}$ id for $i, j=1, \ldots, n-1$. So, we have again a Clifford representation, this time with $m=n-1$ and $k n_{0}=n+1$ for odd $n$. Therefore, by the table above:

- if $n=8 p+1: 8 p+2=k 2^{4 p}$ and hence $p=0, k=2$ and $n=1$;

- if $n=8 p+3: 8 p+4=k 2^{4 p+2}$ and hence $p=0, k=1$ and $n=3$;

- if $n=8 p+5: 8 p+6=k 2^{4 p+3}$, which has no solutions;

- if $n=8 p+7: 8 p+8=k 2^{4 p+3}$ and hence $p=0, k=1$ and $n=7$.

We conclude from this subsection that diagonal decompositions can only occur when the base manifold has dimension $2,3,4,7$ or 8 . (The case $n=1$ is irrelevant, since then $T_{r} M$ has dimension equal to one and no decompositions exist.)

\subsection{The remaining dimensions.}

Case $\mathbf{n}=\mathbf{2}$. In this situation, we have a two-dimensional manifold for which the nullity vector space of the curvature tensor is non-trivial. This implies that the curvature tensor is identically zero and the space is flat.

Conversely, since any tangent sphere bundle of a flat surface $M^{2}(0)$ is a flat threedimensional space, a diagonal decomposition actually exists around each point $(x, u)$ of $T_{r} M^{2}(0)$. Note, however, that we also have $T_{r} M^{2}(0) \simeq M^{2}(0) \times S^{1}(r)$ with $\{x\} \times S^{1}(r) \simeq \pi^{-1}(x)$. So, $T_{r} M^{2}(0)$ also admits a vertical decomposition.

Case $\mathbf{n}=3$. Let $X_{3}$ be the unique unit vector (up to sign) such that $X_{3}{ }^{h}$ is tangent to $M_{1}$ at $(x, u)$. Pick a unit vector $X_{1}$ orthogonal to $X_{3}$ and let $Y_{1}$ be the corresponding unit vector orthogonal to $u$ (i.e., $X_{1}{ }^{h}+\lambda Y_{1}{ }^{t}$ is tangent to $M_{1}$ ). From the comments at the beginning of Subsection 4.3, we know that $(r / 2) R\left(u, Y_{1}\right) X_{3}$ is a unit vector, which is moreover orthogonal to $X_{1}$ and $X_{3}$. So, we obtain an orthonormal basis $\left\{X_{1}, X_{2}, X_{3}\right\}$ by defining $X_{2}$ to be $X_{2}:=(r / 2) R\left(u, Y_{1}\right) X_{3}$. Let $Y_{2}$ be the corresponding unit vector orthogonal to $u$ and $Y_{1}$. (Since each $Y_{i}$ is fixed together with its corresponding $X_{i}$, we will not mention this explicitly anymore in what follows.)

Using the properties of the operators $R\left(u, Y_{1}\right)$ and $R\left(u, Y_{2}\right)$, we then deduce that

$$
\begin{array}{lll}
R\left(u, Y_{1}\right) X_{1}=0, & R\left(u, Y_{1}\right) X_{2}=-\frac{2}{r} X_{3}, & R\left(u, Y_{1}\right) X_{3}=\frac{2}{r} X_{2}, \\
R\left(u, Y_{2}\right) X_{1}=\frac{2}{r} X_{3}, & R\left(u, Y_{2}\right) X_{2}=0, & R\left(u, Y_{2}\right) X_{3}=-\frac{2}{r} X_{1}
\end{array}
$$

and from (13) and (19) it follows that

$$
R\left(Y_{1}, Y_{2}\right) X_{1}=-\frac{2}{r^{2}} X_{2}, \quad R\left(Y_{1}, Y_{2}\right) X_{2}=\frac{2}{r^{2}} X_{1}, \quad R\left(Y_{1}, Y_{2}\right) X_{3}=0 .
$$

Next, we compute $R\left(X_{i}, X_{j}\right) X_{k}, i, j, k=1,2,3$, from the equalities (16) and (22), writing $R\left(u, R\left(X_{i}, X_{j}\right) u\right) X_{k}$ as $\sum g\left(R\left(u, Y_{l}\right) X_{i}, X_{j}\right) R\left(u, Y_{l}\right) X_{k}$ and using (24) and (25). This gives

\begin{tabular}{c|ccc} 
& $X_{1}$ & $X_{2}$ & $X_{3}$ \\
\hline$r^{2} R\left(X_{1}, X_{2}\right)$ & $-A X_{2}$ & $A X_{1}$ & 0 \\
$r^{2} R\left(X_{1}, X_{3}\right)$ & $-C X_{3}$ & 0 & $C X_{1}$ \\
$r^{2} R\left(X_{2}, X_{3}\right)$ & 0 & $-C X_{3}$ & $C X_{3}$
\end{tabular}

where $A=\left(\lambda^{4}-\lambda^{2}+1\right) / \lambda^{2}$ and $C=\left(3 \lambda^{2}+1\right) / \lambda^{2}$. 
Since both $\left\{X_{1}, X_{2}, X_{3}\right\}$ and $\left\{Y_{1}, Y_{2}, u / r\right\}$ are orthonormal bases for $T_{x} M$, there is an orthogonal matrix $Q=\left(q_{i j}\right) \in O(3)$ such that

$$
\left(\begin{array}{c}
X_{1} \\
X_{2} \\
X_{3}
\end{array}\right)=Q\left(\begin{array}{c}
Y_{1} \\
Y_{2} \\
u / r
\end{array}\right) .
$$

Changing $X_{3}$ to $-X_{3}$ if necessary, we may even suppose that $Q \in S O(3)$. Then

$$
\begin{aligned}
R\left(X_{1}, X_{3}\right)= & \left(q_{11} q_{32}-q_{12} q_{31}\right) R\left(Y_{1}, Y_{2}\right)+\frac{q_{11} q_{33}-q_{13} q_{31}}{r} R\left(Y_{1}, u\right) \\
& +\frac{q_{12} q_{33}-q_{13} q_{32}}{r} R\left(Y_{2}, u\right) \\
= & -q_{23} R\left(Y_{1}, Y_{2}\right)-\frac{q_{22}}{r} R\left(u, Y_{1}\right)+\frac{q_{21}}{r} R\left(u, Y_{2}\right) .
\end{aligned}
$$

If we let both sides act on $X_{1}, X_{2}$ and $X_{3}$ and if we use (24), (25) and (26), we find that

$$
q_{21}=-C / 2, \quad q_{22}=0, \quad q_{23}=0 .
$$

Since $Q \in S O(3)$, it follows that $q_{21}{ }^{2}+q_{22}{ }^{2}+q_{23}{ }^{2}=1$ and hence $1=\left(3 \lambda^{2}+1\right) / 2 \lambda^{2}$ or $\lambda^{2}+1=0$, which is a contradiction. Hence, no three-dimensional manifold admits a diagonal decomposition of its tangent sphere bundles at any point.

Case $\mathbf{n}=4$. Let $X_{4}$ be the unique unit vector (up to sign) in the nullity distribution of $R_{x}$. Take two mutually orthogonal unit vectors $X_{1}$ and $X_{2}$ perpendicular to $X_{4}$. Since $(r / 2) R\left(u, Y_{1}\right) X_{2}$ is a unit vector and orthogonal to $X_{1}, X_{2}$ and $X_{4}$, we can define $X_{3}:=(r / 2) R\left(u, Y_{1}\right) X_{2}$. From the properties of the operators $R\left(u, Y_{i}\right)$, $i=1,2,3$, it follows that

\begin{tabular}{c|cccc} 
& $X_{1}$ & $X_{2}$ & $X_{3}$ & $X_{4}$ \\
\hline & & & & \\
$r R\left(u, Y_{1}\right)$ & 0 & $2 X_{3}$ & $-2 X_{2}$ & 0 \\
$r R\left(u, Y_{2}\right)$ & $-2 X_{3}$ & 0 & $2 X_{1}$ & 0 \\
$r R\left(u, Y_{3}\right)$ & $2 X_{2}$ & $-2 X_{1}$ & 0 & 0
\end{tabular}

Next, we decompose $X_{4}$ with respect to the basis $\left\{Y_{1}, Y_{2}, Y_{3}, u / r\right\}$ :

$$
X_{4}=q_{1} Y_{1}+q_{2} Y_{2}+q_{3} Y_{3}+q_{4} \frac{u}{r}, \quad q_{1}^{2}+q_{2}^{2}+q_{3}^{2}+q_{4}^{2}=1 .
$$

Then $R\left(u, X_{4}\right)=q_{1} R\left(u, Y_{1}\right)+q_{2} R\left(u, Y_{2}\right)+q_{3} R\left(u, Y_{3}\right)$. Since $X_{4}$ belongs to the nullity distribution of $R$, this operator vanishes identically. By 27, we must have $q_{1}=q_{2}=q_{3}=0$. Hence, $X_{4}= \pm u / r$. But this is impossible, since $u$ clearly does not belong to the nullity distribution. So, also for four-dimensional manifolds, a diagonal decomposition of its tangent sphere bundles does not exist at any point.

Case $\mathbf{n}=7$. The argument for $n=7$ goes along the same lines as that for $n=3$, but it is more involved technically. Again we start with the unit vector $X_{7}$, uniquely 
determined up to sign, such that $X_{7}{ }^{h}$ is tangent to $M_{1}$, and with an arbitrary unit vector $X_{1}$ orthogonal to $X_{7}$. The unit vector $X_{2}:=(r / 2) R\left(u, Y_{1}\right) X_{7}$ is orthogonal to both $X_{1}$ and $X_{7}$. Then it follows that

$$
\begin{array}{lll}
R\left(u, Y_{1}\right) X_{1}=0, & R\left(u, Y_{1}\right) X_{2}=-\frac{2}{r} X_{7}, & R\left(u, Y_{1}\right) X_{7}=\frac{2}{r} X_{2}, \\
R\left(u, Y_{2}\right) X_{1}=\frac{2}{r} X_{7}, & R\left(u, Y_{2}\right) X_{2}=0, & R\left(u, Y_{2}\right) X_{7}=-\frac{2}{r} X_{1}
\end{array}
$$

Note that $R\left(u, Y_{1}\right)$ and $R\left(u, Y_{2}\right)$ preserve $\operatorname{span}\left\{X_{1}, X_{2}, X_{7}\right\}$, hence by skew-symmetry also its orthogonal complement. Next, take a unit vector $X_{4}$ orthogonal to $X_{1}, X_{2}, X_{7}$ and define the unit vectors $X_{5}:=(r / 2) R\left(u, Y_{2}\right) X_{4}$ and $X_{6}:=$ $(r / 2) R\left(u, Y_{1}\right) X_{4}$. Then $X_{5}$ and $X_{6}$ are already orthogonal to $X_{1}, X_{2}, X_{4}$ and $X_{7}$. Further,

$$
\begin{aligned}
g\left(X_{5}, X_{6}\right) & =\frac{r^{2}}{4} g\left(R\left(u, Y_{2}\right) X_{4}, R\left(u, Y_{1}\right) X_{4}\right) \\
& =-\frac{r^{2}}{4} g\left(R\left(u, Y_{1}\right) R\left(u, Y_{2}\right) X_{4}, X_{4}\right) \\
& =\frac{r^{2}}{4} g\left(R\left(u, Y_{2}\right) R\left(u, Y_{1}\right) X_{4}, X_{4}\right) \quad(\text { by (18) }) \\
& =-\frac{r^{2}}{4} g\left(R\left(u, Y_{1}\right) X_{4}, R\left(u, Y_{2}\right) X_{4}\right) \\
& =-g\left(X_{5}, X_{6}\right)
\end{aligned}
$$

and $X_{5}$ and $X_{6}$ are mutually orthogonal as well. Finally, since $R\left(u, Y_{1}\right) X_{5}$ is orthogonal to $X_{1}, X_{2}, X_{5}, X_{7}$ and

$$
\begin{aligned}
& g\left(R\left(u, Y_{1}\right) X_{5}, X_{4}\right)=-g\left(X_{5}, R\left(u, Y_{1}\right) X_{4}\right)=-\frac{2}{r} g\left(X_{5}, X_{6}\right)=0, \\
& g\left(R\left(u, Y_{1}\right) X_{5}, X_{6}\right)=\frac{r}{2} g\left(R\left(u, Y_{1}\right) X_{5}, R\left(u, Y_{1}\right) X_{4}\right)=\frac{2}{r} g\left(X_{5}, X_{4}\right)=0,
\end{aligned}
$$

we may define $X_{3}:=(r / 2) R\left(u, Y_{1}\right) X_{5}$.

In this way, we have defined an orthonormal basis $\left\{X_{1}, \ldots, X_{7}\right\}$, and the actions of the operators $R\left(u, Y_{i}\right), i=1, \ldots, 6$, can be computed explicitly in this basis using the properties (12), (18) and (23) above. We obtain

\begin{tabular}{c|ccccccc} 
& $X_{1}$ & $X_{2}$ & $X_{3}$ & $X_{4}$ & $X_{5}$ & $X_{6}$ & $X_{7}$ \\
\hline & & & & & & & \\
$r R\left(u, Y_{1}\right)$ & 0 & $-2 X_{7}$ & $-2 X_{5}$ & $2 X_{6}$ & $2 X_{3}$ & $-2 X_{4}$ & $2 X_{2}$ \\
$r R\left(u, Y_{2}\right)$ & $2 X_{7}$ & 0 & $2 X_{6}$ & $2 X_{5}$ & $-2 X_{4}$ & $-2 X_{3}$ & $-2 X_{1}$ \\
$r R\left(u, Y_{3}\right)$ & $2 X_{5}$ & $-2 X_{6}$ & 0 & $-2 X_{7}$ & $-2 X_{1}$ & $2 X_{2}$ & $2 X_{4}$ \\
$r R\left(u, Y_{4}\right)$ & $-2 X_{6}$ & $-2 X_{5}$ & $2 X_{7}$ & 0 & $2 X_{2}$ & $2 X_{1}$ & $-2 X_{3}$ \\
$r R\left(u, Y_{5}\right)$ & $-2 X_{3}$ & $2 X_{4}$ & $2 X_{1}$ & $-2 X_{2}$ & 0 & $-2 X_{7}$ & $2 X_{6}$ \\
$r R\left(u, Y_{6}\right)$ & $2 X_{4}$ & $2 X_{3}$ & $-2 X_{2}$ & $-2 X_{1}$ & $2 X_{7}$ & 0 & $-2 X_{5}$
\end{tabular}


Next, we calculate $R\left(Y_{i}, Y_{j}\right) X_{k}$ from (13) and (19):

\begin{tabular}{c|ccccccc} 
& $X_{1}$ & $X_{2}$ & $X_{3}$ & $X_{4}$ & $X_{5}$ & $X_{6}$ & $X_{7}$ \\
\hline$r^{2} R\left(Y_{1}, Y_{2}\right)$ & $-2 X_{2}$ & $2 X_{1}$ & $2 X_{4}$ & $-2 X_{3}$ & $2 X_{6}$ & $-2 X_{5}$ & 0 \\
$r^{2} R\left(Y_{1}, Y_{3}\right)$ & $-2 X_{3}$ & $-2 X_{4}$ & $2 X_{1}$ & $2 X_{2}$ & 0 & $2 X_{7}$ & $-2 X_{6}$ \\
$r^{2} R\left(Y_{1}, Y_{4}\right)$ & $-2 X_{4}$ & $2 X_{3}$ & $-2 X_{2}$ & $2 X_{1}$ & $2 X_{7}$ & 0 & $-2 X_{5}$ \\
$r^{2} R\left(Y_{1}, Y_{5}\right)$ & $-2 X_{5}$ & $-2 X_{6}$ & 0 & $-2 X_{7}$ & $2 X_{1}$ & $2 X_{2}$ & $2 X_{4}$ \\
$r^{2} R\left(Y_{1}, Y_{6}\right)$ & $-2 X_{6}$ & $2 X_{5}$ & $-2 X_{7}$ & 0 & $-2 X_{2}$ & $2 X_{1}$ & $2 X_{3}$ \\
$r^{2} R\left(Y_{2}, Y_{3}\right)$ & $2 X_{4}$ & $-2 X_{3}$ & $2 X_{2}$ & $-2 X_{1}$ & $2 X_{7}$ & 0 & $-2 X_{5}$ \\
$r^{2} R\left(Y_{2}, Y_{4}\right)$ & $-2 X_{3}$ & $-2 X_{4}$ & $2 X_{1}$ & $2 X_{2}$ & 0 & $-2 X_{7}$ & $2 X_{6}$ \\
$r^{2} R\left(Y_{2}, Y_{5}\right)$ & $2 X_{6}$ & $-2 X_{5}$ & $-2 X_{7}$ & 0 & $2 X_{2}$ & $-2 X_{1}$ & $2 X_{3}$ \\
$r^{2} R\left(Y_{2}, Y_{6}\right)$ & $-2 X_{5}$ & $-2 X_{6}$ & 0 & $2 X_{7}$ & $2 X_{1}$ & $2 X_{2}$ & $-2 X_{4}$ \\
$r^{2} R\left(Y_{3}, Y_{4}\right)$ & $2 X_{2}$ & $-2 X_{1}$ & $-2 X_{4}$ & $2 X_{3}$ & $2 X_{6}$ & $-2 X_{5}$ & 0 \\
$r^{2} R\left(Y_{3}, Y_{5}\right)$ & 0 & $2 X_{7}$ & $-2 X_{5}$ & $-2 X_{6}$ & $2 X_{3}$ & $2 X_{4}$ & $-2 X_{2}$ \\
$r^{2} R\left(Y_{3}, Y_{6}\right)$ & $2 X_{7}$ & 0 & $-2 X_{6}$ & $2 X_{5}$ & $-2 X_{4}$ & $2 X_{3}$ & $-2 X_{1}$ \\
$r^{2} R\left(Y_{4}, Y_{5}\right)$ & $2 X_{7}$ & 0 & $2 X_{6}$ & $-2 X_{5}$ & $2 X_{4}$ & $-2 X_{3}$ & $-2 X_{1}$ \\
$r^{2} R\left(Y_{4}, Y_{6}\right)$ & 0 & $-2 X_{7}$ & $-2 X_{5}$ & $-2 X_{6}$ & $2 X_{3}$ & $2 X_{4}$ & $2 X_{2}$ \\
$r^{2} R\left(Y_{5}, Y_{6}\right)$ & $2 X_{2}$ & $-2 X_{1}$ & $2 X_{4}$ & $-2 X_{3}$ & $-2 X_{6}$ & $2 X_{5}$ & 0
\end{tabular}

Using (16) and (22), we can now compute the curvature components $R\left(X_{i}, X_{j}\right) X_{k}$ for $i, j, k=1, \ldots, 7$ :

\begin{tabular}{c|ccccccc} 
& $X_{1}$ & $X_{2}$ & $X_{3}$ & $X_{4}$ & $X_{5}$ & $X_{6}$ & $X_{7}$ \\
\hline$r^{2} R\left(X_{1}, X_{2}\right)$ & $-A X_{2}$ & $A X_{1}$ & $2 X_{4}$ & $-2 X_{3}$ & $2 X_{6}$ & $-2 X_{5}$ & 0 \\
$r^{2} R\left(X_{1}, X_{3}\right)$ & $-B X_{3}$ & $X_{4}$ & $B X_{1}$ & $-X_{2}$ & 0 & 0 & 0 \\
$r^{2} R\left(X_{1}, X_{4}\right)$ & $-B X_{4}$ & $-X_{3}$ & $X_{2}$ & $B X_{1}$ & 0 & 0 & 0 \\
$r^{2} R\left(X_{1}, X_{5}\right)$ & $-B X_{5}$ & $X_{6}$ & 0 & 0 & $B X_{1}$ & $-X_{2}$ & 0 \\
$r^{2} R\left(X_{1}, X_{6}\right)$ & $-B X_{6}$ & $-X_{5}$ & 0 & 0 & $X_{2}$ & $B X_{1}$ & 0 \\
$r^{2} R\left(X_{1}, X_{7}\right)$ & $-C X_{7}$ & 0 & 0 & 0 & 0 & 0 & $C X_{1}$ \\
$r^{2} R\left(X_{2}, X_{3}\right)$ & $-X_{4}$ & $-B X_{3}$ & $B X_{2}$ & $X_{1}$ & 0 & 0 & 0 \\
$r^{2} R\left(X_{2}, X_{4}\right)$ & $X_{3}$ & $-B X_{4}$ & $-X_{1}$ & $B X_{2}$ & 0 & 0 & 0 \\
$r^{2} R\left(X_{2}, X_{5}\right)$ & $-X_{6}$ & $-B X_{5}$ & 0 & 0 & $B X_{2}$ & $X_{1}$ & 0 \\
$r^{2} R\left(X_{2}, X_{6}\right)$ & $X_{5}$ & $-B X_{6}$ & 0 & 0 & $-X_{1}$ & $B X_{2}$ & 0 \\
$r^{2} R\left(X_{2}, X_{7}\right)$ & 0 & $-C X_{7}$ & 0 & 0 & 0 & 0 & $C X_{2}$ \\
$r^{2} R\left(X_{3}, X_{4}\right)$ & $2 X_{2}$ & $-2 X_{1}$ & $-A X_{4}$ & $A X_{3}$ & $2 X_{6}$ & $-2 X_{5}$ & 0 \\
$r^{2} R\left(X_{3}, X_{5}\right)$ & 0 & 0 & $-B X_{5}$ & $X_{6}$ & $B X_{3}$ & $-X_{4}$ & 0 \\
$r^{2} R\left(X_{3}, X_{6}\right)$ & 0 & 0 & $-B X_{6}$ & $-X_{5}$ & $X_{4}$ & $B X_{3}$ & 0 \\
$r^{2} R\left(X_{3}, X_{7}\right)$ & 0 & 0 & $-C X_{7}$ & 0 & 0 & 0 & $C X_{3}$ \\
$r^{2} R\left(X_{4}, X_{5}\right)$ & 0 & 0 & $-X_{6}$ & $-B X_{5}$ & $B X_{4}$ & $X_{3}$ & 0 \\
$r^{2} R\left(X_{4}, X_{6}\right)$ & 0 & 0 & $X_{5}$ & $-B X_{6}$ & $-X_{3}$ & $B X_{4}$ & 0 \\
$r^{2} R\left(X_{4}, X_{7}\right)$ & 0 & 0 & 0 & $-C X_{7}$ & 0 & 0 & $C X_{4}$ \\
$r^{2} R\left(X_{5}, X_{6}\right)$ & $2 X_{2}$ & $-2 X_{1}$ & $2 X_{4}$ & $-2 X_{3}$ & $-A X_{6}$ & $A X_{5}$ & 0 \\
$r^{2} R\left(X_{5}, X_{7}\right)$ & 0 & 0 & 0 & 0 & $-C X_{7}$ & 0 & $C X_{5}$ \\
$r^{2} R\left(X_{6}, X_{7}\right)$ & 0 & 0 & 0 & 0 & 0 & $-C X_{7}$ & $C X_{6}$
\end{tabular}

where $A=\left(\lambda^{4}-\lambda^{2}+1\right) \lambda^{2}, B=\left(\lambda^{2}+1\right)^{2} / \lambda^{2}$ and $C=\left(3 \lambda^{2}+1\right) / \lambda^{2}$.

We now show that the tables above are incompatible. To see this, we relate the two orthonormal bases $\left\{X_{1}, \ldots, X_{7}\right\}$ and $\left\{Y_{1}, \ldots, Y_{6}, u / r\right\}$ by an orthogonal 
transformation. Let $Q=\left(q_{i j}\right) \in O(7)$ be such that

$$
\left(\begin{array}{c}
X_{1} \\
\vdots \\
X_{7}
\end{array}\right)=Q\left(\begin{array}{c}
Y_{1} \\
\vdots \\
Y_{6} \\
u / r
\end{array}\right) .
$$

Putting $Q_{k l}^{i j}:=q_{i k} q_{j l}-q_{i l} q_{j k}$, we then have the equality

$$
R\left(X_{i}, X_{j}\right)=\sum_{k<l=1}^{6} Q_{k l}^{i j} R\left(Y_{k}, Y_{l}\right)+\sum_{k=1}^{6}\left(Q_{k 7}^{i j} / r\right) R\left(Y_{k}, u\right) .
$$

So,

$$
\begin{aligned}
2 & =r^{2} g\left(R\left(X_{1}, X_{2}\right) X_{3}, X_{4}\right) \\
& =\sum_{k<l=1}^{6} r^{2} Q_{k l}^{12} g\left(R\left(Y_{k}, Y_{l}\right) X_{3}, X_{4}\right)+\sum_{k=1}^{6} r Q_{k 7}^{12} g\left(R\left(Y_{k}, u\right) X_{3}, X_{4}\right) \\
& =2\left(Q_{12}^{12}-Q_{34}^{12}+Q_{56}^{12}\right)
\end{aligned}
$$

and

$$
2=r^{2} g\left(R\left(X_{1}, X_{2}\right) X_{5}, X_{6}\right)=2\left(Q_{12}^{12}+Q_{34}^{12}-Q_{56}^{12}\right) .
$$

This implies that $Q_{12}^{12}=1$. Now, using the Cauchy-Schwarz inequality and the fact that $Q$ is orthogonal, we find that

$$
\begin{aligned}
1 & =Q_{12}^{12}=q_{11} q_{22}-q_{12} q_{21}=\left(q_{11}, q_{12}\right) \cdot\left(q_{22},-q_{21}\right) \\
& \leq \sqrt{{q_{11}{ }^{2}+q_{12}}^{2}} \sqrt{{q_{21}{ }^{2}+q_{22}}^{2}} \leq \sqrt{q_{11^{2}}+\cdots+q_{17}{ }^{2}} \sqrt{q_{21}^{2}+\cdots+q_{27}^{2}}=1 .
\end{aligned}
$$

Hence, all the inequalities must be equalities. In particular, we have $q_{13}=\cdots=$ $q_{17}=q_{23}=\cdots=q_{27}=0$ and consequently

$$
X_{1}=\cos \theta_{1} Y_{1}+\sin \theta_{1} Y_{2}, \quad X_{2}=\epsilon_{1}\left(-\sin \theta_{1} Y_{1}+\cos \theta_{1} Y_{2}\right)
$$

where $\epsilon_{1}= \pm 1$ and $\theta_{1}$ is some real number. In a similar way, we can show that $Q_{34}^{34}=Q_{56}^{56}=1$ and that

$$
\begin{aligned}
& X_{3}=\cos \theta_{2} Y_{3}+\sin \theta_{2} Y_{4}, \quad X_{4}=\epsilon_{2}\left(-\sin \theta_{2} Y_{3}+\cos \theta_{2} Y_{4}\right), \\
& X_{5}=\cos \theta_{3} Y_{5}+\sin \theta_{3} Y_{6}, \quad X_{6}=\epsilon_{3}\left(-\sin \theta_{3} Y_{5}+\cos \theta_{3} Y_{6}\right) .
\end{aligned}
$$

As a consequence, we also have $X_{7}=\epsilon u / r, \epsilon= \pm 1$. Using the tables above, we find that

$$
\begin{aligned}
0 & =r^{2} R\left(X_{1}, X_{7}\right) X_{3}=-\epsilon\left(\cos \theta_{1} r R\left(u, Y_{1}\right) X_{3}+\sin \theta_{1} r R\left(u, Y_{2}\right) X_{3}\right) \\
& =2 \epsilon\left(\cos \theta_{1} X_{5}-\sin \theta_{1} X_{6}\right),
\end{aligned}
$$

which gives a contradiction. So, also seven-dimensional manifolds cannot have a diagonal decomposition for their tangent sphere bundles at any point.

Case $\mathbf{n}=8$. This case is treated as the case $n=4$, but the appropriate choice for the basis $\left\{X_{1}, \ldots, X_{8}\right\}$ requires a little more care. Let $X_{8}$ be the unique unit vector (up to sign) in the nullity distribution of $R_{x}$ and take two arbitrary unit vectors $X_{1}$ and $X_{2}$ that are mutually orthogonal and perpendicular to $X_{8}$. As before, we 
define $X_{3}:=(r / 2) R\left(u, Y_{1}\right) X_{2}$, which is a unit vector orthogonal to $X_{1}, X_{2}$ and $X_{8}$. It follows that $R\left(u, Y_{i}\right) X_{8}=0$ for $i=1,2,3$, and

$$
\begin{array}{lll}
R\left(u, Y_{1}\right) X_{1}=0, & R\left(u, Y_{1}\right) X_{2}=\frac{2}{r} X_{3}, & R\left(u, Y_{1}\right) X_{3}=-\frac{2}{r} X_{2}, \\
R\left(u, Y_{2}\right) X_{1}=-\frac{2}{r} X_{3}, & R\left(u, Y_{2}\right) X_{2}=0, & R\left(u, Y_{2}\right) X_{3}=\frac{2}{r} X_{1}, \\
R\left(u, Y_{3}\right) X_{1}=\frac{2}{r} X_{2}, & R\left(u, Y_{3}\right) X_{2}=-\frac{2}{r} X_{1}, & R\left(u, Y_{3}\right) X_{3}=0 .
\end{array}
$$

Because they are skew-symmetric, the operators $R\left(u, Y_{1}\right), R\left(u, Y_{2}\right)$ and $R\left(u, Y_{3}\right)$ also preserve $W=\left\{X_{1}, X_{2}, X_{3}, X_{8}\right\}^{\perp}$, and on this space they anti-commute by (18). It is easy to check that the operator $\left(r^{3} / 8\right) R\left(u, Y_{1}\right) R\left(u, Y_{2}\right) R\left(u, Y_{3}\right)$ is symmetric on $W$ and that it squares to the identity on $W$. Hence, it has a basis of eigenvectors corresponding to the eigenvalues +1 and -1 . Let $X_{4}$ be a unit vector in $W$ such that $r^{3} R\left(u, Y_{1}\right) R\left(u, Y_{2}\right) R\left(u, Y_{3}\right) X_{4}=8 \epsilon X_{4}$ where $\epsilon= \pm 1$, and define

$$
X_{5}:=\frac{r}{2} R\left(u, Y_{1}\right) X_{4}, \quad X_{6}:=\frac{r}{2} R\left(u, Y_{2}\right) X_{4}, \quad X_{7}:=\frac{r}{2} R\left(u, Y_{3}\right) X_{4} .
$$

Clearly, $X_{5}, X_{6}$ and $X_{7}$ are unit vectors orthogonal to $X_{1}, X_{2}, X_{3}, X_{4}$ and $X_{8}$. A computation similar to (28) shows that they are also orthogonal to one another. So, we have an orthonormal basis $\left\{X_{1}, \ldots, X_{8}\right\}$ for $T_{x} M$. It is now possible to compute explicitly the action of $R\left(u, Y_{i}\right), i=1, \ldots, 7$, from the condition (18). We get

\begin{tabular}{c|cccccccc} 
& $X_{1}$ & $X_{2}$ & $X_{3}$ & $X_{4}$ & $X_{5}$ & $X_{6}$ & $X_{7}$ & $X_{8}$ \\
\hline & & & & & & & & \\
$r R\left(u, Y_{1}\right)$ & 0 & $2 X_{3}$ & $-2 X_{2}$ & $2 X_{5}$ & $-2 X_{4}$ & $-2 \epsilon X_{7}$ & $2 \epsilon X_{6}$ & 0 \\
$r R\left(u, Y_{2}\right)$ & $-2 X_{3}$ & 0 & $2 X_{1}$ & $2 X_{6}$ & $2 \epsilon X_{7}$ & $-2 X_{4}$ & $-2 \epsilon X_{5}$ & 0 \\
$r R\left(u, Y_{3}\right)$ & $2 X_{2}$ & $-2 X_{1}$ & 0 & $2 X_{7}$ & $-2 \epsilon X_{6}$ & $2 \epsilon X_{5}$ & $-2 X_{4}$ & 0 \\
$r R\left(u, Y_{4}\right)$ & $-2 X_{5}$ & $-2 X_{6}$ & $-2 X_{7}$ & 0 & $2 X_{1}$ & $2 X_{2}$ & $2 X_{3}$ & 0 \\
$r R\left(u, Y_{5}\right)$ & $2 X_{4}$ & $-2 \epsilon X_{7}$ & $2 \epsilon X_{6}$ & $-2 X_{1}$ & 0 & $-2 \epsilon X_{3}$ & $2 \epsilon X_{2}$ & 0 \\
$r R\left(u, Y_{6}\right)$ & $2 \epsilon X_{7}$ & $2 X_{4}$ & $-2 \epsilon X_{5}$ & $-2 X_{2}$ & $2 \epsilon X_{3}$ & 0 & $-2 \epsilon X_{1}$ & 0 \\
$r R\left(u, Y_{7}\right)$ & $-2 \epsilon X_{6}$ & $2 \epsilon X_{5}$ & $2 X_{4}$ & $-2 X_{3}$ & $-2 \epsilon X_{2}$ & $2 \epsilon X_{1}$ & 0 & 0
\end{tabular}

Next, decompose $X_{8}$ with respect to the basis $\left\{Y_{1}, \ldots, Y_{7}, u / r\right\}$ :

$$
X_{8}=q_{1} Y_{1}+\cdots+q_{7} Y_{7}+q_{8} \frac{u}{r}, \quad q_{1}^{2}+\cdots+q_{8}{ }^{2}=1 .
$$

Since $X_{8}$ belongs to the nullity distribution of the curvature, we have

$$
0=R\left(u, X_{8}\right)=\sum_{i=1}^{7} q_{i} R\left(u, Y_{i}\right)
$$

and from the table above we deduce $q_{1}=\cdots=q_{7}=0$. Hence, $X_{8}= \pm u / r$, but this is impossible since $u$ does not belong to the nullity distribution. So, also in the eight-dimensional case, a diagonal decomposition of the tangent sphere bundles does not exist at even a single point.

Remark 3. The operator $r^{3} R\left(u, Y_{1}\right) R\left(u, Y_{2}\right) R\left(u, Y_{3}\right)$ acts as $8 \epsilon$ id on the vector space spanned by $X_{4}, X_{5}, X_{6}$ and $X_{7}$, as is seen easily from the previous table. The two different cases, $\epsilon=+1$ and $\epsilon=-1$, correspond to the two non-equivalent irreducible Clifford representations of the seven-dimensional Clifford algebra. 


\section{VERTICAL DECOMPOSITION}

Now, we suppose that we have a vertical decomposition $T_{r} M \simeq M_{1} \times M_{2}$ such that $V T_{r} M_{(x, u)} \subset T_{(x, u)} M_{1}$ everywhere. In this situation, if $(x, u) \in M_{1} \times\{q\}$ for some $q \in M_{2}$, then $\pi^{-1}(x) \subset M_{1} \times\{q\}$. Consequently, we have $M_{1} \times\{q\}=$ $\pi^{-1}\left(\pi\left(M_{1} \times\{q\}\right)\right)$. So, the leaves $M_{1} \times\{q\}$, corresponding to the product, project under $\pi$ to a foliation $\mathbf{L}_{1}$ on $(M, g)$ and $\pi^{-1}\left(\mathbf{L}_{1}\right)=\left\{M_{1} \times\{q\}, q \in M_{2}\right\}$. Let $L_{1}$ be the distribution on $M$ tangent to $\mathbf{L}_{1}$. Define the distribution $L_{2}$ to be the orthogonal distribution to $L_{1}$ on $M$. Then

$$
T_{(x, u)}\left(M_{1} \times\{q\}\right)=V T_{r} M_{(x, u)} \oplus h\left(L_{1 x}\right), \quad T_{(x, u)}\left(\{p\} \times M_{2}\right)=h\left(L_{2 x}\right)
$$

where $h$ denotes the horizontal lift.

If $X$ and $Y$ are vector fields on $M$ tangent to $L_{1}$ and $U, V$ are tangent to $L_{2}$, then $X^{h}, Y^{h}$ are tangent to $M_{1}$ and $U^{h}, V^{h}$ are tangent to $M_{2}$. Because of the product structure, we have that $\bar{\nabla}_{X^{h}} Y^{h}$ and $\bar{\nabla}_{U^{h}} X^{h}$ are tangent to $M_{1}$ and $\bar{\nabla}_{U^{h}} V^{h}$ and $\bar{\nabla}_{X^{h}} U^{h}$ are tangent to $M_{2}$. Using the expressions (11) for $\bar{\nabla}$, this means that

- $\nabla_{X} Y$ and $\nabla_{U} X$ are sections of $L_{1}$ : so, $L_{1}$ is totally geodesic and even totally parallel;

- $\nabla_{U} V$ and $\nabla_{X} U$ are sections of $L_{2}$ : so, also $L_{2}$ is totally geodesic and totally parallel (in particular, $L_{2}$ is integrable with associated foliation $\mathbf{L}_{2}$ );

- $R(U, V) u=R(X, U) u=0$ : so, $L_{2}$ is contained in the nullity distribution of the curvature. The leaves of $\mathbf{L}_{2}$ are therefore flat.

These properties imply that $\mathbf{L}_{1}$ and $\mathbf{L}_{2}$ consist of the leaves of a local Riemannian product $M \simeq M^{\prime} \times \mathbb{R}^{k}$ where $k=\operatorname{dim} L_{2} \leq n$ (see [8]).

Suppose conversely that $M^{n}$ is locally isometric to $M^{\prime} \times \mathbb{R}^{k}$ for $1 \leq k \leq n$. This gives rise to two foliations on $M^{n}: \mathbf{L}_{1}=\left\{M^{\prime} \times\{v\}, v \in \mathbb{R}^{k}\right\}$ and $\mathbf{L}_{2}=\left\{\{p\} \times \mathbb{R}^{k}, p \in\right.$ $\left.M^{\prime}\right\}$. Define two complementary distributions $\tilde{L}_{1}$ and $\tilde{L}_{2}$ on $T_{r} M$ by

$$
\tilde{L}_{1}=V T_{r} M \oplus h\left(T M^{\prime}\right), \quad \tilde{L}_{2}=h\left(T \mathbb{R}^{k}\right) .
$$

It is easily checked using (1) that $\tilde{L}_{1}$ and $\tilde{L}_{2}$ are totally geodesic and totally parallel complementary distributions. Hence, the leaves of their corresponding foliations $\tilde{\mathbf{L}}_{1}$ and $\tilde{\mathbf{L}}_{2}$ are actually the leaves of a local Riemannian product. In particular, note that $\tilde{\mathbf{L}}_{1}=\left\{\pi^{-1}\left(M^{\prime} \times\{v\}\right), v \in \mathbb{R}^{k}\right\}$. So, $T_{r} M$ is indeed locally reducible.

\section{Global Results}

We continue with the notation of the previous section. In order to derive results concerning the global reducibility of $\left(T_{r} M, g_{S}\right)$, we will exploit the relationship between the foliations $\mathbf{L}_{1}$ and $\mathbf{L}_{2}$ of $(M, g)$ and the foliations $\tilde{\mathbf{L}}_{1}$ and $\tilde{\mathbf{L}}_{2}$ of $\left(T_{r} M, g_{S}\right)$ in the case of a vertical decomposition. We have already remarked that $\mathbf{L}_{1}$ and $\tilde{\mathbf{L}}_{1}$ determine each other reciprocally by $\mathbf{L}_{1}=\pi\left(\tilde{\mathbf{L}}_{1}\right)$ and $\tilde{\mathbf{L}}_{1}=\pi^{-1} \mathbf{L}_{1}$. The relationship between the foliations $\mathbf{L}_{2}$ and $\tilde{\mathbf{L}}_{2}$ is not so straightforward. We still have $\mathbf{L}_{2}=$ $\pi\left(\tilde{\mathbf{L}}_{2}\right)$, but determining $\tilde{\mathbf{L}}_{2}$ from $\mathbf{L}_{2}$ requires a little more care. To construct the leaf $\tilde{S}$ of $\tilde{\mathbf{L}}_{2}$ through a point $(x, u) \in T_{r} M$, consider all the curves in the leaf $S$ of $\mathbf{L}_{2}$ starting at $x \in M$. Then, $\tilde{S}$ consists of all end-points of the horizontal lifts of these curves starting at $(x, u)$. We call $\tilde{S}$ the horizontal lift of $S$ through $(x, u)$. Since $\tilde{S}$ is everywhere horizontal, the map $\pi: \tilde{S} \rightarrow S$ is a local isometry and $\tilde{S}$ is a Riemannian covering of $S$. When $S$ is simply connected, $\tilde{S}$ and $S$ are globally isometric and, in particular, one-to-one. 
With these comments in mind, we now proceed to the proof of the Global Theorem. So, we suppose that $\operatorname{dim} M \geq 3$ and that $\left(T_{r} M, g_{S}\right)$ is isometric to a global Riemannian product $\left(M_{1}, g_{1}\right) \times\left(M_{2}, g_{2}\right)$. Since $\operatorname{dim} M \geq 3$, this is a vertical decomposition and $V T_{r} M$ is tangent to one of the factors, say $M_{1}$. Consider $M_{1}$ and $M_{2}$ as submanifolds of $T_{r} M$ (i.e., choose one leaf of each of the foliations $\tilde{\mathbf{L}}_{1}$ and $\tilde{\mathbf{L}}_{2}$ ) and define the submanifolds $M^{\prime}:=\pi\left(M_{1}\right)$ and $F:=\pi\left(M_{2}\right)$ of $M$. From the local considerations in the previous section, we know that $(M, g)$ is locally isometric to the Riemannian product $M^{\prime} \times F$ and that $F$ is flat.

We show that there is a one-to-one correspondence between $M$ and $M^{\prime} \times F$. Take a point $x \in M$ and consider an arbitrary vector $u \in T_{x} M$ of length $r$. Through $(x, u) \in T_{r} M$, there is a unique leaf $\tilde{S}_{1 u}$ of $\tilde{\mathbf{L}}_{1}$ and a unique leaf $\tilde{S}_{2 u}$ of $\tilde{\mathbf{L}}_{2}$. Because of the product structure on $T_{r} M, \tilde{S}_{1 u}$ cuts $M_{2}$ in a unique point $\tilde{q}_{u} \in M_{2}$ and $\tilde{S}_{2 u}$ cuts $M_{1}$ in a unique point $\tilde{p}_{u} \in M_{1}$. Put $p_{u}:=\pi\left(\tilde{p}_{u}\right) \in M^{\prime}$ and $q_{u}:=\pi\left(\tilde{q}_{u}\right) \in F$. We claim that the correspondence $M \rightarrow M^{\prime} \times F: x \mapsto\left(p_{u}, q_{u}\right)$ is well-defined, i.e., independent of the choice of the tangent vector $u$. To see this, take another vector $v \in T_{x} M$ of length $r$. Since $\pi(x, u)=x=\pi(x, v)$, the leaf $\tilde{S}_{1 u}$ of $\tilde{\mathbf{L}}_{1}$ contains both $(x, u)$ and $(x, v)$; so we have $\tilde{S}_{1 u}=\tilde{S}_{1 v}, \tilde{q}_{u}=\tilde{q}_{v}$ and $q_{u}=q_{v}$. The unique leaf $\tilde{S}_{2 v}$ of $\tilde{\mathbf{L}}_{2}$ through $(x, v)$ is different from $\tilde{S}_{2 u}$. However, both are horizontal lifts of $S_{2 x}=\pi\left(\tilde{S}_{2 u}\right)$. So, if $\tilde{\gamma}_{u}(t)=(x(t), u(t))$ is a curve in $\tilde{S}_{2 u}$ such that $\tilde{\gamma}_{u}(0)=(x, u)$ and $\tilde{\gamma}_{u}(1)=\tilde{p}_{u} \in M_{1}$, then $\gamma=\pi \circ \tilde{\gamma}_{u}$ runs from $x$ to $p_{u} \in M$ in $S_{2 x}$. Denote by $\tilde{\gamma}_{v}$ the horizontal lift of $\gamma$ starting at $(x, v)$. Clearly, $\tilde{\gamma}_{v}$ lies in $\tilde{S}_{2 v}$ and ends at $\tilde{p}_{v} \in M_{1}$. Hence, $p_{v}=\pi\left(\tilde{p}_{v}\right)=\pi\left(\tilde{\gamma}_{v}(1)\right)=\gamma(1)=p_{u}$.

On the other hand, starting from a couple $(p, q) \in M^{\prime} \times F$, we find the corresponding point $x \in M$ as $x=\pi(\tilde{p}, \tilde{q})$ for some $\tilde{p} \in M_{1}$ with $\pi(\tilde{p})=p$ and the unique $\tilde{q} \in M_{2}$ with $\pi(\tilde{q})=q$. Via an argument as above, one shows that $x$ does not depend on the choice of $\tilde{p}$ and that the map $(p, q) \mapsto x$ defined in this way is the inverse of the map $x \mapsto\left(p_{u}, q_{u}\right)$.

Next, we note that the correspondence $M \rightarrow M^{\prime} \times F: x \mapsto\left(p_{u}, q_{u}\right)$ is defined so as to respect the local product structure. In particular, the metric $g$ on $M$ corresponds to the product metric of $M^{\prime} \times F$, and the first statement is proved.

Conversely, suppose that $(M, g)$ is the global product space $\left(M^{\prime}, g^{\prime}\right) \times\left(F, g_{0}\right)$. By choosing a leaf of both product foliations, one can consider $M^{\prime}$ and $F$ as submanifolds of $M$. Let $x_{0}$ be their intersection point and choose a vector $u_{0} \in T_{x_{0}} M$ of length $r$. Define $M_{1}$ as the inverse image of $M^{\prime}$ under the projection $\pi$ and $M_{2}$ as the horizontal lift of $F$ through $\left(x_{0}, u_{0}\right)$. Since we suppose $F$ to be simply connected, $M_{2}$ is isometric to the flat space $\left(F, g_{0}\right)$ and $M_{1}$ and $M_{2}$ have $\left(x_{0}, u_{0}\right)$ as unique intersection point.

We show that there is a one-to-one correspondence between $T_{r} M$ and $M_{1} \times M_{2}$. Take $(x, u) \in T_{r} M$ and denote by $S_{1}$ the unique leaf of $\mathbf{L}_{1}$ on $M$ through $x$ and by $S_{2}$ the unique leaf of $\mathbf{L}_{2}$ on $M$ through $x$. Then, the leaf $\tilde{S}_{1}$ of $\tilde{\mathbf{L}}_{1}$ through $(x, u)$ is given by $\pi^{-1}\left(S_{1}\right)$ and the leaf $\tilde{S}_{2}$ of $\tilde{\mathbf{L}}_{2}$ through $(x, u)$ is the horizontal lift of $S_{2}$ through this point. $\tilde{S}_{1}$ cuts $M_{2}$ in a unique point $\tilde{q}$ with $\pi(\tilde{q})=S_{1} \cap F$, and $\tilde{S}_{2}$ cuts $M_{1}$ in a unique point $\tilde{p}$ with $\pi(\tilde{p})=S_{2} \cap M^{\prime}$. (Note that the simply connectedness of $F$ is essential to ensure uniqueness.) Clearly, the correspondence $T_{r} M \rightarrow M_{1} \times M_{2}:(x, u) \mapsto(\tilde{p}, \tilde{q})$ is well-defined and it is not difficult to construct its inverse. Since this correspondence also respects the local product structure, the metric $g_{S}$ on $T_{r} M$ corresponds to the product metric on $M_{1} \times M_{2}$. This completes the proof of the Global Theorem. 
Remark 4. The proof of the Global Theorem continues to hold when $n=2$ for the case of a vertical global decomposition of $\left(T_{r} M, g_{S}\right)$. Clearly, the base manifold is then flat. That we need the simply connectedness of the flat factor can be seen from the example of a two-dimensional flat cone $C$. The vertical and horizontal distributions on $T_{r} C$ are both integrable, and locally their integral manifolds are the leaves of the local product foliation on $T_{r} C$. If it were a global decomposition, every maximal integral manifold of the horizontal distribution would intersect every vertical fiber exactly once and it would be isometric to $C$ under the natural projection $\pi$. This would define a global parallelization of $C$, contrary to the fact that its full holonomy group is non-trivial.

\section{REFERENCES}

[1] M. F. Atiyah, R. Bott and A. Shapiro, Clifford modules, Topology 3, Suppl. 1 (1964), 3-38. MR 29:5250

[2] J. Berndt, E. Boeckx, P. Nagy and L. Vanhecke, Geodesics on the unit tangent bundle, preprint, 2001.

[3] J. Berndt, F. Tricerri and L. Vanhecke, Generalized Heisenberg groups and Damek-Ricci harmonic spaces, Lecture Notes in Math. 1598, Springer-Verlag, Berlin, Heidelberg, New York, 1995. MR 97a:53068

[4] E. Boeckx and G. Calvaruso, When is the unit tangent sphere bundle semi-symmetric?, preprint, 2002.

[5] E. Boeckx and L. Vanhecke, Characteristic reflections on unit tangent sphere bundles, Houston J. Math. 23 (1997), 427-448. MR 2000e:53052

[6] E. Boeckx and L. Vanhecke, Curvature homogeneous unit tangent sphere bundles, Publ. Math. Debrecen 53 (1998), 389-413. MR 2000d:53080

[7] E. Boeckx and L. Vanhecke, Harmonic and minimal vector fields on tangent and unit tangent bundles, Differential Geom. Appl. 13 (2000), 77-93. MR 2001f:53138

[8] G. de Rham, Sur la reductibilité d'un espace de Riemann, Comment. Math. Helv. 26 (1952), 328-344. MR 14:584a

[9] O. Kowalski and M. Sekizawa, On tangent sphere bundles with small or large constant radius, Ann. Global Anal. Geom. 18 (2000), 207-219. MR 2001i:53049

[10] O. Kowalski, M. Sekizawa and Z. Vlášek, Can tangent sphere bundles over Riemannian manifolds have strictly positive sectional curvature?, in: Global Differential Geometry: The Mathematical Legacy of Alfred Gray (eds. M. Fernández, J. A. Wolf), Contemp. Math. 288, Amer. Math. Soc., Providence, RI, 2001, 110-118. MR 2002i:53047

Department of Mathematics, Katholieke Universiteit Leuven, Celestijnenlaan 200B, 3001 Leuven, Belgium

E-mail address: eric.boeckx@wis.kuleuven.ac.be 